\title{
Visual search of cyclic spatio-temporal events
}

\author{
Jacques GAUTIER $^{\mathrm{a}}$, Paule-Annick DAVOINE ${ }^{\mathrm{b}}$, and Claire CUNTY ${ }^{\mathrm{b}}$ \\ ${ }^{a}$ Univ. Grenoble Alpes, CNRS, Grenoble INP, LIG, F-38000 Grenoble, France, 700 avenue centrale, 38401 Saint Martin-d'Hères, \\ France,Jacques.Gautier@imag.fr,Paule-Annick.Davoine@imag.fr \\ ${ }^{b}$ UMR EVS/IRG Lyon II, 5 avenue Pierre Mendès-France, 69500 Bron, France, Claire.Cunty@univ-lyon2.fr
}

\begin{abstract}
The analysis of spatio-temporal events, and especially of relationships between their different dimensions (space-time-thematic attributes), can be done with geovisualization interfaces. But few geovisualization tools integrate the cyclic dimension of spatio-temporal event series (natural events or social events). Time Coil and Time Wave diagrams represent both the linear time and the cyclic time. By introducing a cyclic temporal scale, these diagrams may highlight the cyclic characteristics of spatio-temporal events. However, the settable cyclic temporal scales are limited to usual durations like days or months. Because of that, these diagrams cannot be used to visualize cyclic events, which reappear with an unusual period, and don't allow to make a visual search of cyclic events. Also, they don't give the possibility to identify the relationships between the cyclic behavior of the events and their spatial features, and more especially to identify localised cyclic events. The lack of possibilities to represent the cyclic time, outside of the temporal diagram of multi-view geovisualization interfaces, limits the analysis of relationships between the cyclic reappearance of events and their other dimensions. In this paper, we propose a method and a geovisualization tool, based on the extension of Time Coil and Time Wave, to provide a visual search of cyclic events, by allowing to set any possible duration to the diagram's cyclic temporal scale. We also propose a symbology approach to push the representation of the cyclic time into the map, in order to improve the analysis of relationships between space and the cyclic behavior of events.
\end{abstract}

Keywords: geovisualization, spatio-temporal events, multi-view interfaces, visual analysis, cyclic reappearance, temporal diagrams, dimensional relationships

\section{Introduction}

For many years, the geovisualization research has focused on the development of frameworks for representing spatial-temporal dynamics. Several cartographic concepts, or methods which use computer sciences, have been proposed in order to integrate the temporal dimension into geovisualization tools (Davoine and al. 2015): maps collection, animated maps, interactive and dynamic maps, using of $3 \mathrm{D}$ interfaces, ... One widely used method is the multi-view interface, which consists in representing each dimension of the spatio-temporal information (spatial, temporal, thematic) in a dedicated frame, synchronized with the others (Kraak 2010 ; Moisuc and al. 2005). These multi-view interfaces allow to make a visual exploration of spatio-temporal data, and provide an analysis of the relationships between the dimensional characteristics of spatio-temporal events.

In these multi-view interfaces, the temporal dimension is represented by temporal diagrams, which are mainly timelines or timewheels (Edsall and Peuquet 1997). However, spatio-temporal events can have both linear and cyclic temporal characteristics. To take into account these characteristics, some authors have proposed temporal diagrams, which associate linear and cyclic aspects of time: "Time Coil" (Edsall and Peuquet 1997); "Time Wave" (Li 2010).

Integrated in multi-view interfaces, these diagrams can be used for analyzing relationships between the spatial or the thematic dimension, and the temporal characteristics of an event series, which has a known cyclic behavior. For example, the influence of the spatial location on the daily moment of occurrence of an event, in a cyclic temporal scale of one day. However, these diagrams are not designed to provide a visual search of the existence of cyclic phenomena, which could show a reappearance period different than usual durations like day, month or year. Also, these diagrams don't provide a method to analyze relationships between the thematic or especially the spatial dimension, and the cyclic period of reappearance of events. In this paper, we first present what we define as a "spatio-temporal phenomenon" and a "spatio-temporal event", as well as the concept of "temporality" that we use to describe the temporal dimension of a spatio-temporal event. Then we present the main approaches to integrate time into geovisualization interfaces. Next, we talk about the advantages of diagrams which include linear and cyclic aspects of time, like Time Coil and Time Wave diagrams, and the limits of these diagrams. Finally, we propose an extension of the use of these diagrams into geovisualization tools. The aim of this proposition is to improve the analysis of cyclic spatio-temporal events, and notably the relationships between their cyclic behavior and their thematic or spatial dimension.

\section{Visualization of spatio-temporal events}

\subsection{How we model spatio-temporal phenomena}

A spatio-temporal phenomenon implies a change in space along time (Haddad 2009). Several propositions have 
been made in order to model the change in space. This part is not intended to make a complete state of the art of all these propositions, or to propose a new modeling approach. Its aim is to define the terms we will use and to precise the structure of the data we use in our proposition. A spatio-temporal phenomenon can be defined in two ways (Haddad 2009): on one hand, a phenomenon can be described as a modification, which affects permanent spatial objects. On the other hand, it can be described as an distinct entity, bounded in time and space, which is at the origin of this modification. A forest fire can be modeled as the destruction of parts of a permanent spatial object "forest", or as a distinct spatio-temporal entity, with a temporal and a spatial extent, which could cause a modification of the object "forest".

These spatio-temporal entities can be considered as something which has an impact on permanent spatial entities (Grenon and Smith 2004). They have a beginning, an end, and an impact on the life trajectory of timeless entities (Mathian and Sanders 2014). A river's flooding is a spatio-temporal entity, which impacts a timeless river. Another approach of the modeling of a phenomenon by a distinct spatio-temporal entity is described by Reitsma (2004). This approach only represents the events, and does not represent their impact on the evolution of permanent spatial objects, which are not taken into account. A rainfall can be represented as a spatio-temporal entity, with a start date, an end date, and a spatial extent, without representing the spatial objects impacted by the rainfall.

The datasets we dispose are formed by event series, where each event has a start date, sometimes a duration, a spatial location, sometimes a spatial shape, and thematic attributes. We don't have any information about objects they could impact. In accordance with these data, we consider spatio-temporal phenomena as global spatiotemporal entities. Their instances are called "spatiotemporal events", which are also spatio-temporal entities. The rain is a global spatio- temporal phenomenon, and one rainfall in particular is a spatio-temporal event. Spatio-temporal events have a start date, a duration, an end date, thematic attributes, a spatial location, and a spatial shape. The timeless spatial objects which could be impacted by these events are not taken into account in our model.

\subsection{The three dimensions of the spatio-temporal information}

Peuquet (1994) described the spatio-temporal information through three dimensions according to the "triad" model: the spatial, the temporal and the thematic dimension (Fig. 1).

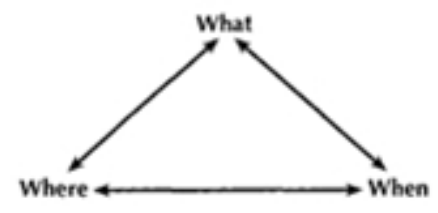

Fig. 1. Triad model (Source: Peuquet 1994)
The analysis of spatio-temporal events has to focus on each dimension, and on the relationships between these dimensions:

- What ? + Where ? $\rightarrow$

When ? When does an event of type 'B' occur in this area?

- What? + When ? $\rightarrow$

Where? Where does an event of type 'B' occur at 9 p.m.?

- When ? + Where ? $\rightarrow$

What? What type of event does occur at 9 p.m. in this area?

\subsection{How to integrate time into geovisualization interfaces}

In order to analyze changes in space along time, several methods have been developed to integrate time into cartographic representations. Davoine (and al. 2015) described four main approaches to integrate time into geovisualization tools: the animated maps, where real time is represented by the time of the animation; the maps collection (small multiple maps), where each map represents the state of a spatial zone at a specific temporal moment; the space-time cube proposed by the Time Geography (Hägerstrand 1973), where time is represented by a third graphic axis, and the multi-view geovisualization interfaces (Fig. 2).

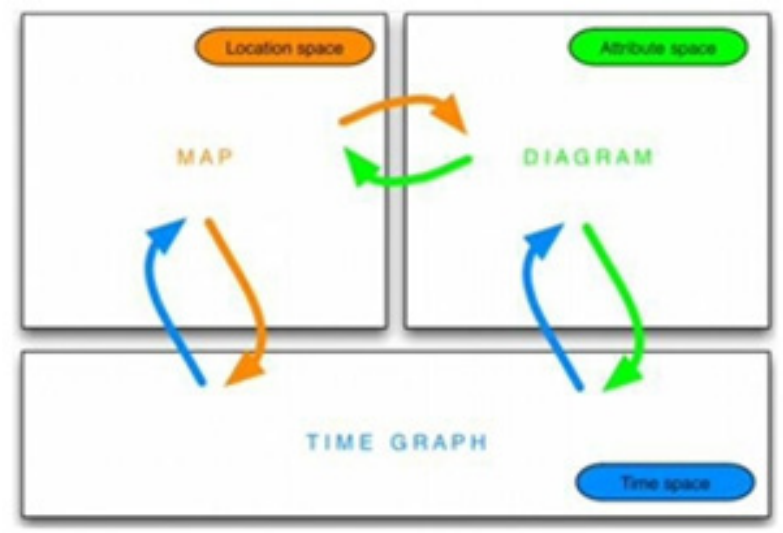

Fig. 2. Multi-view interface (Source: Kraak and al. 2010)

These last interfaces follow the concept of the orthogonality of spatio-temporal information described by Kraak (and al. 2010) and Davoine and Moisuc (and al. 2005). They are structured into three (or more) synchronized windows. Each of them represents one dimension of the spatio-temporal information. The spatial dimension is usually represented by a map, the thematic dimension by a thematic graph, and the temporal dimension by a temporal diagram. Each window acts as a visual query tool in order to analyze the data according to one specific dimensional criterion. The analysis, provided by this visualization structure and the dimensional querying, allows to answer to the questions introduced by the triad model (What? Where? When?).

Two main tools are usually used as temporal diagrams (Edsall and al. 1997): timelines and timewheels. The timeline is a representation of the linear aspect of time, in 
whose events are represented by graphical objects along a graduated linear axis: one point on the diagram represents one temporal moment in an absolute linear temporal scale. Timewheels represent the cyclic aspect of time, where time evolution is a repetition of a temporal period (day, week, month, year). The timewheel describes a circle representing an instance of the considered time period (one day, one week, one month, one year). One point on the circle curve represents one temporal moment in a relative cyclic temporal scale.

Timelines are used to visualize the temporal dimension of events according to an absolute and linear vision of time (one event occurs at the 5th of November 1605). Timewheels are used to visualize them according to a relative and cyclic time scale (one event appends at 9 am, in the morning, during the beginning of the year). Edsall and Peuquet (1997) proposed to combine the two diagrams into the same time visualization tool, in order to select spatio-temporal data according to the two conceptualizations of time (Fig. 3).

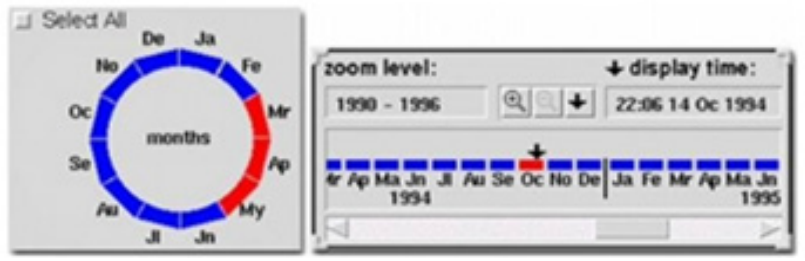

Fig. 3. TEMPEST interface (Source: Edsall and Peuquet 1997)

\section{Visualization of cyclic reappearance of spatio- temporal events}

3.1 Return period, period of reappearance and cyclic phenomenon

Hewitt (1983), Allen (1983), DiBiase (and al. 1992), and MacEachren (1994) proposed to describe the temporal dimension of a spatio-temporal event with six temporalities (Fig. 4):

Three temporalities describe the event itself

- the moment of occurrence of the event

- the duration of the event

- the return-period of the event

three other temporalities describe temporal relationships between several events

- the chronological order of the occurrence of several events

- the chronological space

- the synchronization of the occurrence of several events

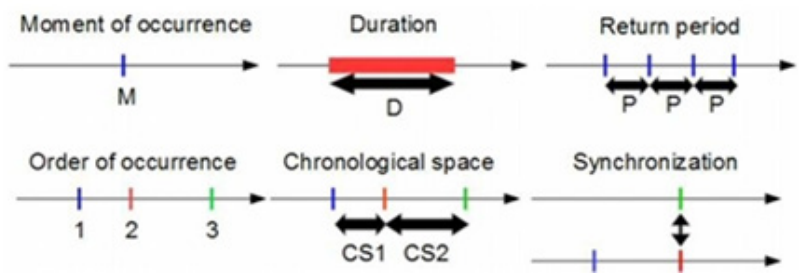

Fig. 4. Temporalities (Source: Hewitt 1983; Allen 1983; DiBiase and al. 1992; MacEachren 1994)
The meaning of the temporality « return period " is ambiguous. In the field of risk sociology, for instance, the return period is the probabilistic duration between two disasters (Arnaud 2009). The return period is not related to the "frequency" of a phenomenon, which is, in the field of risk sociology, the number of occurrences of a phenomenon during a specified duration (Arnaud 2009).

We focus here on what we could call the "period of reappearance" of a cyclic phenomenon, which describes the duration between the moment of occurrence of two instances of a cyclic phenomenon. The term « cyclic phenomenon» is also ambiguous, because a real cycle implies the reappearance of strictly identical events in strictly identical conditions, with a strictly regular period of reappearance, which is rarely observed. We call here « cyclic phenomenon» the regular reappearance of events considered as similar. The similarity of these events is arbitrary and depends on the criteria we use to classify the events. We also call « cyclic phenomenon» a reappearance, which doesn't possess a strictly regular period of reappearance. A phenomenon, which appears every morning but not at the same precise hour, will be considered as cyclic. We also consider as a cyclic phenomenon, a reappearance with a practically regular period of appearance but with periods, for which the event doesn't appear: a phenomenon which appears every morning expect for one day is considered as cyclic.

We try here to visualize the cyclic behavior of a phenomenon, and to identify the value of its period of reappearance.

\subsection{Visualization of cyclic phenomena on temporal diagrams}

The visualization of time in multi-view geovisualization tools by timelines and timewheels allows to interrogate the temporal dimension of events according to a linear or a cyclic scale, and to directly visualize temporalities of events:

- the moment (When) is represented by the position of a graphical object on the diagram

- the duration (How long) is represented by the length of a graphical object on the diagram

- the frequency (How often) is represented by the number of graphical objects on a portion of the diagram

- the chronological order (In which order) and the synchronization is represented by the distribution of graphical objects on the diagram

- the chronological space is represented by the distance between graphical objects on the diagram

To visualize a cyclic reappearance of events in timelines, the user has to identify a regular length between each graphical object corresponding to the same phenomenon (Fig. 5). The same identification of a regular length between graphical objects is necessary in timewheels, even if timewheels integrate the cyclic aspect of time evolution (Fig. 5). 


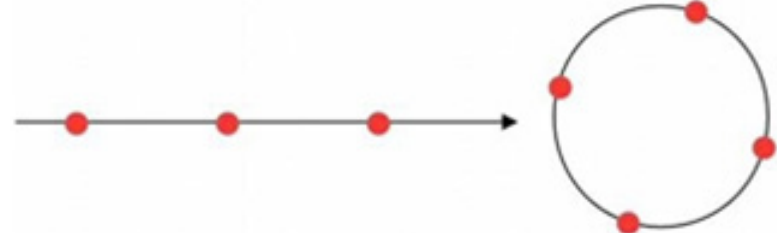

Fig. 5. Visualizing cyclic reappearance in timelines and timewheels

Other methods have been developed to integrate linear and cyclic aspects of time into a single temporal diagram. Edsall and Peuquet (1997) proposed a temporal diagram called "Time Coil", represented by a helical curve created along a linear axis (Fig. 6). This axis represents the linear aspect of time, and the curve's revolution represents the cyclic aspect of time.

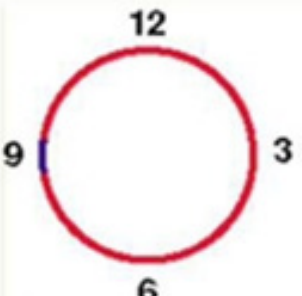

6

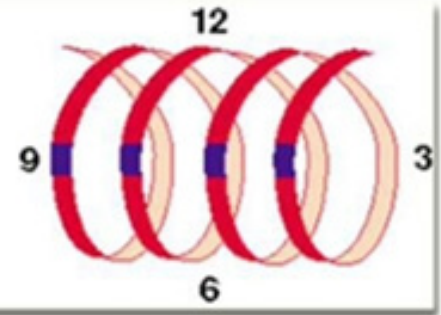

Fig. 6. Time Coil (Source: Edsall and Peuquet 1997)
Li (2010) proposed a similar temporal diagram called "Time Wave", which can be seen as a plane version of Time Coil (Fig. 7). The linear aspect of time is represented by a linear axis, and the cyclic aspect of time is represented by waves formed by the diagram's curve.

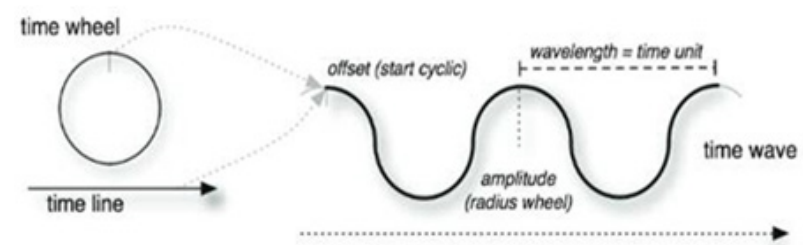

Fig. 7. Time Wave (Source: Li 2010)

These diagrams allow to visualize the temporal dimension of events according to the two aspects of time (linear and absolute, cyclic and relative) in the same graph. The user can visualize when an event appears according to an absolute and linear temporal scale (the 5 th of November 1605), and according to a relative and cyclic temporal scale (the morning, the end of the year, ...) in the same tool. These diagrams are interactive and allow to change the duration of the cyclic temporal scale, in order to analyze the temporal dimension of one event according to several cyclic temporal scales (Does an event occur at the beginning of a day? of a month? ...).

These diagrams allow also to directly visualize a cyclic reappearance of events. If the duration of the cyclic temporal scale is equal to the period of reappearance of a cyclic phenomenon, the corresponding graphical objects will form an alignment, parallel to the linear axis of the diagram. By adding the possibility to use different cyclic temporal scales, cyclic reappearance of events with different periods can be visualized on these diagrams.

\subsection{Limits of Time Wave and Time Coil diagrams}

\subsubsection{These tools are not designed to identify cyclic events}

Even if these diagrams allow to directly visualize a cyclic reappearance of events, this possibility is limited by the values that can be set to the cyclic temporal scale. To illustrate this assertion, we will focus on the Time Wave diagram.

The visualization of time by timelines and timewheels does not always fit to analyze spatio-temporal events, because many phenomena have both linear and cyclic characteristics, for example in the fields of meteorology, finance and physics ( $\mathrm{Li}$ 2010). The Time Wave diagram has been created in order to be integrated into a multiview geovisualization tool, and to provide an analysis of spatio-temporal events which include the two aspects of time. However, the objective of this diagram is to provide an analysis of temporal characteristics of cyclic phenomena, but not to provide a way to identify if a phenomenon is cyclic or not.

In one case study presented in Time Wave works, the analysis focused on a daily cyclic phenomenon: the reach of the daily maximum temperature in several weather stations. The user had to identify, for each station, the daily moment of occurrence of the event according to a daily temporal scale. Fig. 8 shows the moments of occurrence of the daily maximum temperature for two stations. Each station is represented by a specific color.

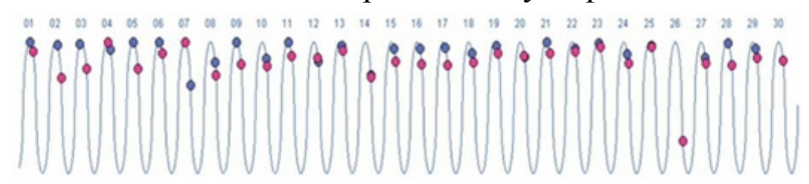

Fig. 8. Time Wave case study (Source: Li 2010)

The phenomenon was already considered as cyclic. The goal of the analysis was not to identify if the phenomenon was cyclic or not, or if space had an influence on the value of its period of reappearance. The aim of the analysis was to identify the irregularities along time of the daily moment of occurrence of this phenomenon in a relative temporal scale, and the influence of space on these irregularities.

This search of the existence of a cyclic reappearance of events can not be easily done with these diagrams. Indeed, according to Hornsby and Egenhofer (2002), the frequent representations of the cycles of time are close of the rhythms of nature or the rhythms in everyday life. Periodic scales, including the settable scales in Time Wave, are created from usual time periods corresponding to cycles we naturally observe or use like days, or months. The period of reappearance which can be visualized in these diagrams are limited to these usual durations. The analysis of the existence of cyclic phenomena is limited. 
3.3.2 Difficulty to analyze relationships between the spatial dimension and the cyclic behavior of events

In a multi-view interface, the symbology applied to events allows to highlight a dimensional characteristic of one event in other windows that the dedicated one. For example, it allows to represent a thematic value in spatial and temporal windows, or the spatial location in the temporal window.

This visualization helps to identify relationships between the dimensions of a phenomenon. In our case, it could be interesting to identify the influence of spatial dimension, like the area of occurrence, on the existence of a cyclic behavior, as well as on the duration of the corresponding period of reappearance.

Time Wave proposes to represent events on the temporal diagram according to their spatial location, which can help to identify the influence of space on the moment of occurrence according to a cyclic temporal scale. This can be obtained by creating spatial areas to classify events according to their belonging to these areas. This classification of events could allow to highlight new alignments of similar graphical objects on the temporal diagram, which will correspond to cyclic events located in a single area.

However, creating areas, in which we could observe local cyclic phenomena, is difficult if we don't know or suspect by advance where these local cycles are. The analysis of relationships between space and the period of reappearance of cyclic phenomena seems to be hard or almost impossible to do with this method.

\section{Proposition}

We propose to extend the Time Wave model. Our objective is to provide a method of spatio-temporal events analysis, which allows a visual search of cyclic reappearance of events. We also want to improve the identification of relationships between the cyclic behavior of events and space. The method we propose is based on a multi-view interface. The temporal dimension is represented by a diagram with a Time Wave structure.

\subsection{Provide a visual search of cyclic events, with a dynamic and gradual change of the cyclic temporal scale}

The direct visualization of cyclic events could be possible with a Time Wave diagram, but this analysis is limited by the settable cyclic temporal scales. By adding the possibility to set any duration to the cyclic temporal scale, we allow to identify any cyclic phenomena, whatever the value of its period of reappearance.

In Fig. 9, we represent a temporal diagram integrating the structure of Time Wave. On this diagram, we put graphical objects, which correspond to events we have created, and which can be classified in two types: "A" and " $\mathrm{B}$ ". In order to highlight the " $\mathrm{A}$ " events, we use color to represent the type of the events: " $A$ " events are colored red, "B" events are colored blue. Other types of symbology can be set in our prototype.
In Fig. 9:a, the duration of the cyclic temporal scale, called "P", is equal to 200 days. From Fig. 9:b to Fig. 9:f, "P" is gradually modified from 53 to 45 days. The values of "P" are respectively equal to $53,50,48,46$, and 45 days. In Fig. 9:b, the "A" events are forming a sinusoidal line on the diagram. From Fig. 9:b to Fig. 9:f, the more "P" is close to 45 days, the more the red objects tend to form a straight line parallel to the linear axis of this diagram. In Fig. 9:f, when $P$ is equal to 45 days, the red objects form an alignment parallel to the diagram's linear axis. We can observe that " $A$ " events are cyclic with a period of reappearance of 45 days.

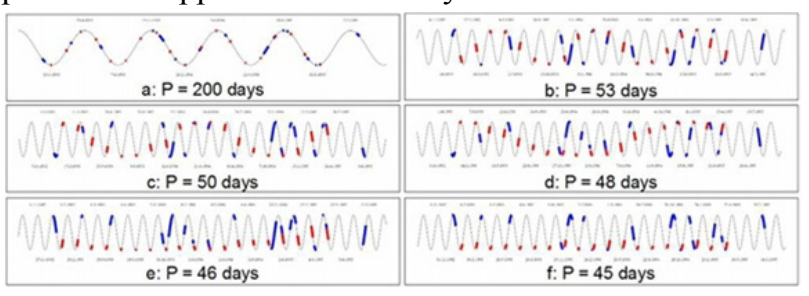

Fig. 9. Visual search of cyclic events

By changing the value of the cyclic temporal scale, we can visually explore the data in order to find cyclic phenomena. This method could also allow to identify relationships between cyclic behavior of events, and the dimensional characteristic used to classify our events. Here we can see a relationship between the type of the events, and their cyclic reappearance.

\subsection{An analysis of relationships between space and the cyclic behavior of events, with the representation of the cyclic time into the map}

A symbology, applied to events, highlights those that have a particular thematic or spatial characteristic, into the temporal diagram. The use of the symbology, done in parallel with a dynamic modification of the cyclic temporal scale, could provide a visual way to analyze relationships between these dimensional characteristics and the cyclic behavior of events. However, we said before that a visual search of local cyclic events is still difficult with a representation of space into the temporal diagram, because we don't know where local cycles can be found. Yet, this analysis of relationships between space and the cyclic behavior of events can be done, through the representation of the cyclic aspect of time into the map.

To represent spatial dimension, we propose to use a map, which represents, at the same time, all the events, which occur during the temporal period covered by the temporal diagram. The events, which occur before or after this period, shall not be displayed on the map. If we change the period covered by the diagram, other events will be displayed on the map.

Since we represent, into the same map, some events which do not share the same moment of occurrence, we can use semiologic rules to represent them according to their temporal dimension. We propose to give the possibility to represent events according to their moment of occurrence related to the cyclic temporal scale, which is used in the temporal diagram. For this purpose, we can 
use different visual variables, like color or direction. The spatial objects, which share the same representation, correspond to objects which are located on the same cyclic section of the temporal diagram. On this diagram, these events form an alignment parallel to the linear axis. If spatial objects, located in the same area, share the same representation, the corresponding events can be considered as instances of a local cyclic phenomenon. The temporal diagram and the map are synchronized, and a modification of the cyclic temporal scale of the temporal diagram will change the representation of the events on the map.

In Fig. 10, 11 and 12, we represent fictional events that happened around the city of Grenoble (France). In Fig. 10 and 11, we use color to represent events according to their moment of occurrence: In this case, the start and the end of the cyclic temporal scale correspond to the color blue, and the middle of the temporal scale corresponds to the color red. The event takes a color between blue and red according to its moment of occurrence related to the cyclic temporal scale.

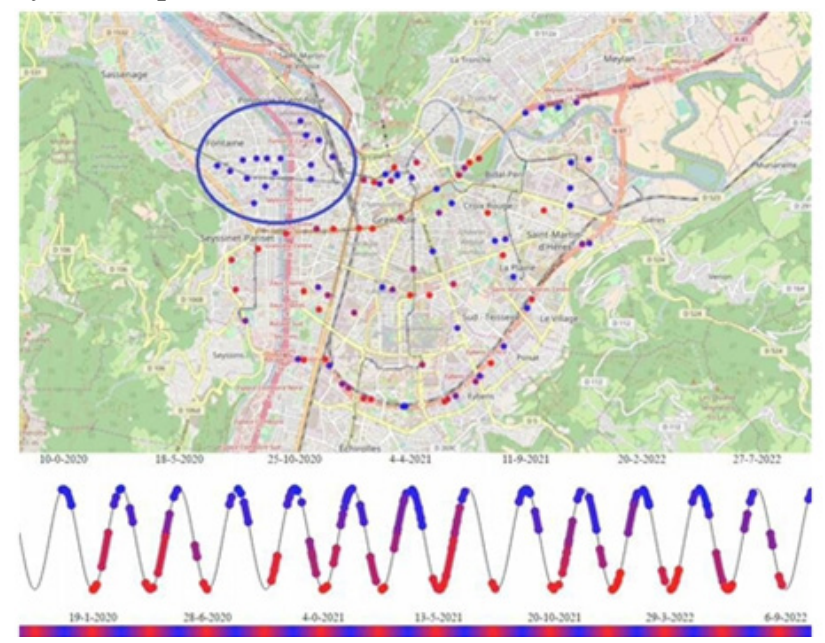

Fig. 10. Cyclic temporal scale equal to 80 days

In Fig. 10, the cyclic temporal scale is equal to 80 days. The map show that the events located in the north west of the city share the same visual representation (they are colored blue).

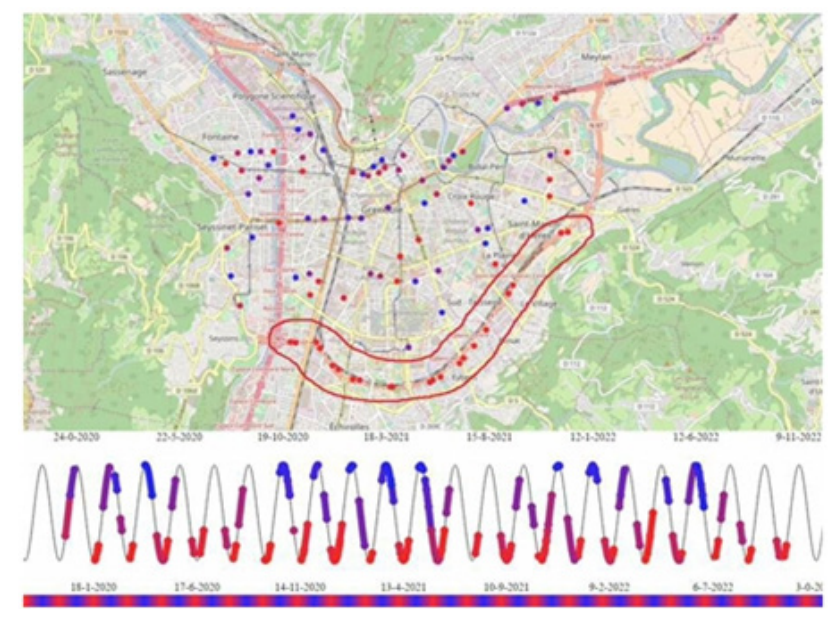

Fig. 11. Cyclic temporal scale equal to 50 days
In Fig. 11, the cyclic temporal scale is equal to 50 days. The events located along the south ring-road share the same visual representation (they are colored red).

In Fig. 12, we classify the events according to their spatial location. The events, which appear in the north west, are colored blue and the events, which appear on the south ring road, are colored red. The other events are colored grey. In Fig. 12:a, the cyclic temporal scale is equal to 80 days, and the blue events are aligned in the temporal diagram. In Fig. 12:b, the cyclic temporal scale is equal to 50 days, and the red events also form an alignment. These alignments confirm that the two groups of events, which shared the same representation in Fig. 10 and 11 , are cyclic.
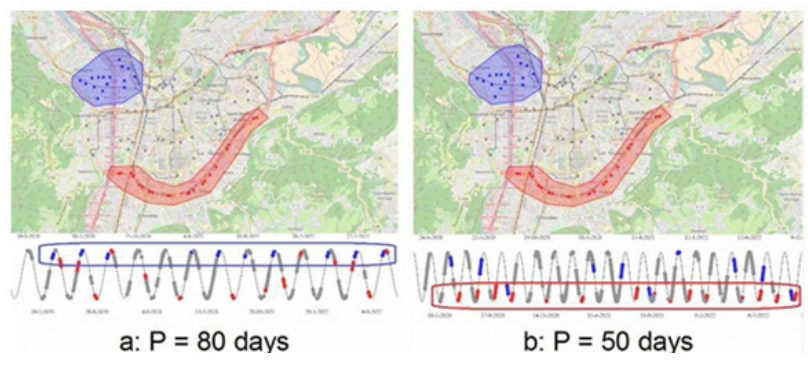

Fig. 12. Spatial classification of events

By changing the value of the cyclic temporal scale, the user can visually explore the data in order to identify, on the map, the formation of groups of spatial objects, which share the same representation. Through this visual search of local cyclic events, we can do a visual analysis of relationships between the cyclic reappearance of events and their spatial dimension. We can, for instance, identify where are located cyclic events, or analyze the influence of space on the duration of the period of reappearance of cyclic phenomena.

\section{Conclusion}

We have specified and implemented a methodology to provide a visual search of the existence of cyclic spatiotemporal events in evenemential dataset. This visual search could be done complementary to mathematical analysis of the period of reappearance of these events. The possibility to represent the cyclic aspect of time into the cartographic interface, through the use of semiologic rules, could improve the analysis of relationships between the spatial dimension of events and their cyclic characteristics. Especially, the influence of space on the period reappearance of cyclic phenomena. However, even if we are using real data to implement our prototype, the effectiveness of our proposition in cyclic phenomena analysis has not been tested yet in a specific and concrete context with users. But the fields of application could be vast and cover any field where cyclic events can be observed, and where the influence of space on the reappearance of events has a great importance. We already work to apply this proposition on natural hazards data and car accident data.

\section{Acknowledgements}

The funding of this work by the Region AuvergneRhône-Alpes is gratefully acknowledged. 


\section{References}

Allen J.-F. (1983) Maintaining knowledge about temporal intervals. Communication of the ACM, 21(11):832-843.

Arnaud A., Davoine P.-A. (2009) Cartographie des temporalités dans le domaine des risques. Comité Français de Cartographie, №202, December 2009

Davoine P.-A., Mathian H., Saint-Marc C., Kaddouri L., Blaise J.-Y. (2015) The Visual Representations of Territorial Dynamics: Retrospective and Input from New Computing Environments. In Proceedings of 27th International Cartographic Conference, 16th General Assembly, Rio de Janeiro, Brazil.

DiBiase D., MacEachren A.-M., Krygier J.-B., Reeves C. (1992) Animation and the role of map design in scientific visualization.

Cartography and Geographic Information Systems, 19(4): 201-214.

Edsall R., Kraak M.-J., MacEachren A.M., Peuquet D.J. (1997) Assessing the effectiveness of temporal legends in environmental visualization, pages 667-685 GIS/LIS, Cincinnati.

Edsall R., Peuquet D. (1997). A graphical user interface for the integration of time into GIS. Research paper of The Apoala Project. Pennsylvania.

Grenon P., and Smith, B. (2004) SNAP and SPAN: Towards Dynamic Spatial Ontology. Spatial Cognition and Computation, Vol. 4 (1), 2004, pp. $69-103$.

Haddad H. (2009) Une approche pour supporter l'analyse qualitative des suites d'actions dans un environnement géographique virtuel et dynamique. L'analyse « What-if » comme exemple. PhD Thesis, Université Laval, Québec, Canada.

Hägerstrand T. (1970) What about people in regional science ? In Papers of the Regional Science Association, $\mathrm{N}^{\circ} 24$, pp. 7-21.

Hewitt K. (1983) The Idea of Calamity in a Technocratic Age. In K. Hewitt, The risks and hazards series, pages 3-32. Allen and Unwin Inc., Boston.

Hornsby L., Egenhofer M.-J. (2002) Modeling Moving Objects over Multiple Granularities. Annals of Mathematics and Artificial Intelligence, 36 :177194.

Kraak M.-J., Ormeling F. (2010) Cartography, Visualization of spatial data. Routledge.

Li X. (2010) The Time wave in time space. A visual exploration environment for spatio-temporal data. ITC Printing Department, University of Twente.

MacEachren A.-M. (1994) Time as a cartographic variable. In Unwin D.J., Hearnshaw H.M., Wiley, Visualization in Geographic Information Systems, pages 115--130.

Mathian H., Sanders L. (2014) Objets géographiques et processus de changement, approches spatiotemporelles.ISTE Editions, London.

Moisuc B., Davoine P.-A., Gensel J., Martin H. (2005) Design of Spatio-Temporal Information Systems for
Natural Risk Management with an Object-Based Knowledge Representation Approach, Geomatica, Vol. 59, No. 4.

Peuquet, D. (1994). It s About Time A Conceptual Framework for the Representation of Temporal Dynamics in Geographic Information Systems. Annals of the Association of American Geographers, Vol. 84(3), 1994, pp. 441 - 461

Reitsma, F. (2004) A New Geographic Process Data Model. PhD thesis, Faculty of the Graduate School of the University of Maryland, College Park, 2004 